\title{
Risk Factors Associated with the Halo Phenomenon after Lumbar Fusion Surgery and its Clinical Significance
}

\author{
Ho-Joong Kim, Sul-Gee Kim, Hwan-Mo Lee, Hak-Sun Kim, Eun-Su Moon, \\ Jin-Oh Park, Nam-Heon Seol, Seong-Hwan Moon \\ Department of Orthopedic Surgery, Yonsei University Medical College, Seoul, Korea
}

\begin{abstract}
Study Design: Retrospective study.
Purpose: First, to examine the association between bone mineral density (BMD) and the halo phenomenon, and second, to investigate risk factors predisposing to the halo phenomenon and its correlation with clinical outcomes.

Overview of Literature: The few in vivo studies regarding the relationship between pedicle screw stability and BMD have shown conflicting results.

Methods: Forty-four female patients who underwent spine fusion surgery due to spinal stenosis were included in this study. The halo phenomenon and fusion state were evaluated through plain radiographs performed immediately after surgery and through the final outpatient follow-up examination. BMD, osteoarthritis grade in the hip and knee joints, and surgical outcome were also evaluated.

Results: BMD was not related to the halo phenomenon, but age, absence of osteoarthritis in the knee, and non-union state were found to be significant risk factors for the halo phenomenon. However, the radiological halo phenomenon did not cor relate with clinical outcome (visual analogue scale for back pain and leg pain).

Conclusions: The halo phenomenon is a simple phenomenon that can develop during follow-up after pedicle screw fixation. It does not influence clinical outcomes, and thus it is thought that hydroxyapatite coating screws, expandable screws, cement augmentation, and additional surgeries are not required, if their purpose is to prevent the halo phenomenon.
\end{abstract}

Key Words: Halo phenomenon, Pedicle screw, Bone mineral density

\section{Introduction}

Pedicle screw fixation is a basic method for thoracolumbar fixation. Pedicle screws provide stability, but they do not sacrifice normal spinal segments by fixating a single segment. Furthermore, firm fixation shows strong resistance to loading from all directions ${ }^{1-4}$. However, despite such advantages, implant failure has been reported with the use of pedicle screws, including screw bending, breakage, and loosening (radiolucency in the bone-screw interface). According to a previous study, the incidence of screw loosening and breakage were between $0.6 \%$ and $25 \%$ and between $0.6 \%$ and $11 \%$, respectively ${ }^{5}$. Presently, due to the increase in pedicle screw diameter and shank tapering, the incidence of bending and breakage are $\operatorname{low}^{6,7}$. However, problems with internal fixators related to screw loosening continue to occur ${ }^{8}$.

The stability of pedicle screws is primarily dependent on the bone-screw interface, and loosening is induced by cyclic caudo-cephalad toggling acting on the bone-screw interface when axial compression is transmitted through metal plates or rods ${ }^{6,9}$. In cases where pedicle screws cannot be fixated adequately to the vertebral body through the pedicles, loosening of screws may occur. Therefore, evaluation of the stability of the bone-screw interface is important in order to

Corresponding author: Seong-Hwan Moon, MD

Department of Orthopedic Surgery, Yonsei University College of Medicine

134 Seodaemunku, Shinchondong, Seoul, Korea

Tel: +82-2-2228-2188, Fax: +82-2-363-1139, E-mail: shmoon@yuhs.ac 
predict the development of screw loosening. If surgeons could predict the development of screw loosening and anticipate the potential risks of loss of correction due to it, supplementary augmentation could be used, and it could be managed more carefully in the postoperative stage.

Previous in vitro studies have shown that the bone mineral density (BMD) of the lumbar vertebral body has an effect on the stability of the pedicle screws ${ }^{10-13}$. Wittenberg et al. ${ }^{14}$ studied cadaveric lumbar vertebral bodies and reported that, in physiological loading, screw loosening developed in vertebrae with BMD lower than $74 \pm 17 \mathrm{mg} / \mathrm{cc}$. Yamagata et al. ${ }^{15}$, have reported that there is a positive correlation between the BMD and the pullout strength of pedicle screws. However, there have been few studies regarding the association between BMD and the halo phenomenon. Therefore, the purpose of our study was first to examine the association between BMD and the halo phenomenon, and second to investigate the risk factors for the halo phenomenon, and its correlation with clinical outcomes.

\section{Materials and Methods}

This study was performed on 44 female patients older than 50 years of age who visited our hospital for symptomatic spinal stenosis. The mean patient age at the time of surgery was 61.3 years (range, 50 79 years). Twenty-six patients had only spinal stenosis, and 18 patients had spinal stenosis with spondylolisthesis. All patients underwent laminectomy, facetectomy, and segmental spinal instrumentation using the Diapason pedicle screw system and posterolateral fusion using an auto-bone graft. Resected laminar bone or autoiliac bone was used as an autogenous bone graft. Thirty-four cases were monosegmental, and ten cases were bisegmental. The postoperative follow-up period ranged from 24 months to 43 months.

The halo phenomenon and fusion state were evaluated through plain radiographs performed immediately after surgery and through the final outpatient follow-up examination. Radiographic fusion patterns were classified into four grades by Lenke et al. ${ }^{16}$, definite solid, possibly solid, probably not solid, and definitely not solid. The grades "definite" and "possibly solid" were considered fusion states, and the criteria of "probably not solid" and "definitely not solid" were designated as non-union. In addition, for the hip and knee joint, the grade of osteoarthritis was evaluated by taking plain radiographs at the time of surgery according to the Kellgren-Lawrence grade ${ }^{17}$. Radiological analysis was performed by two orthopedic surgeons who were unaware of this study.

In all patients, BMD was measured prior to surgery using dual-energy x-ray absorptiometry (DEXA) (Hologic QDR4500 \& Hologic Delthi, Waltham, MA, USA). Measurements were obtained in the proximal femur and lumbar vertebral body. Kim \& Kim's criteria and visual analogue scale (VAS) were used for assessment of clinical results, both back pain and leg pain ${ }^{18,19}$.

\section{Statistical analysis}

All data were analyzed using the SPSS 12.0.1 statistics package (SPSS Inc., Chicago, IL, USA). The Mann-Whitney test was used for comparing BMD between the halo phenomenon and non-halo phenomenon groups.

In halo phenomenon cases with more than 2 pedicle screws, the odds ratio for each risk factor was calculated using multivariate logistic regression analysis. This categorical halo phenomenon (more than 2 pedicle screws or not) was used as a dependent variable. Age, weight, BMD, VAS for back pain, VAS for leg pain, and fusion state were assigned as continuous independent co-variates. Menopause, osteoarthritis in the knee, and fusion state (non-union) were assigned as categorical, independent co-variates.

The correlation between the radiological halo phenomenon and the VAS for leg and back pain was analyzed using a chi-square test. A p-value $<0.05$ was considered statistically significant.

\section{Results}

\section{Bone mineral density}

In terms of BMD, fifteen patients (36\%) had osteoporosis (T score <-2.5), and 21 patients (47\%) had osteopenia, which was defined as a T score between -1.0 and -2.5 . Eight patients (18\%) showed normal BMD. In all patients, the mean (SD) BMD value of the lumbar vertebra was 0.839 $(0.207) \mathrm{g} / \mathrm{cm}^{2}$, and that of the proximal femur was 0.404 $(0.150) \mathrm{g} / \mathrm{cm}^{2}$.

\section{Clinical assessment}

The clinical results were satisfactory (good or fair) in 
forty patients (90\%), based on Kim and Kim's criteria. In the assessment of VAS for leg pain, the mean postoperative VAS at last follow-up was much lower than the mean preoperative VAS; the mean postoperative VAS (mm) was 8 , while the mean preoperative VAS ( $\mathrm{mm}$ ) was 78 . The VAS for back pain was also improved from 43 preoperatively to 34 postoperatively.

\section{Radiological assessment}

In the assessment of osteoarthritis of the knee and hip joints, 19 patients had osteoarthritis of the knee joint (Kellgren-Lawrence grade II, III, or IV), and no patients had osteoarthritis of the hip joint. At last follow-up, radiological assessment showed "definitely" or "probably solid fusion mass" in 37 patients $(84 \%)$. There was "probably not solid fusion mass" without implant failure in 7 patients (16\%). The halo phenomenon was observed in at least one pedicle screw in 21 patients (48\%), among which 13 patients had the phenomenon in more than 2 pedicle screws and 3 patients had it in all pedicle screws (Figs. 1 and 2). There were no screw or rod breakages, and only one patient developed progressive kyphosis due to loosening without screw breakage.
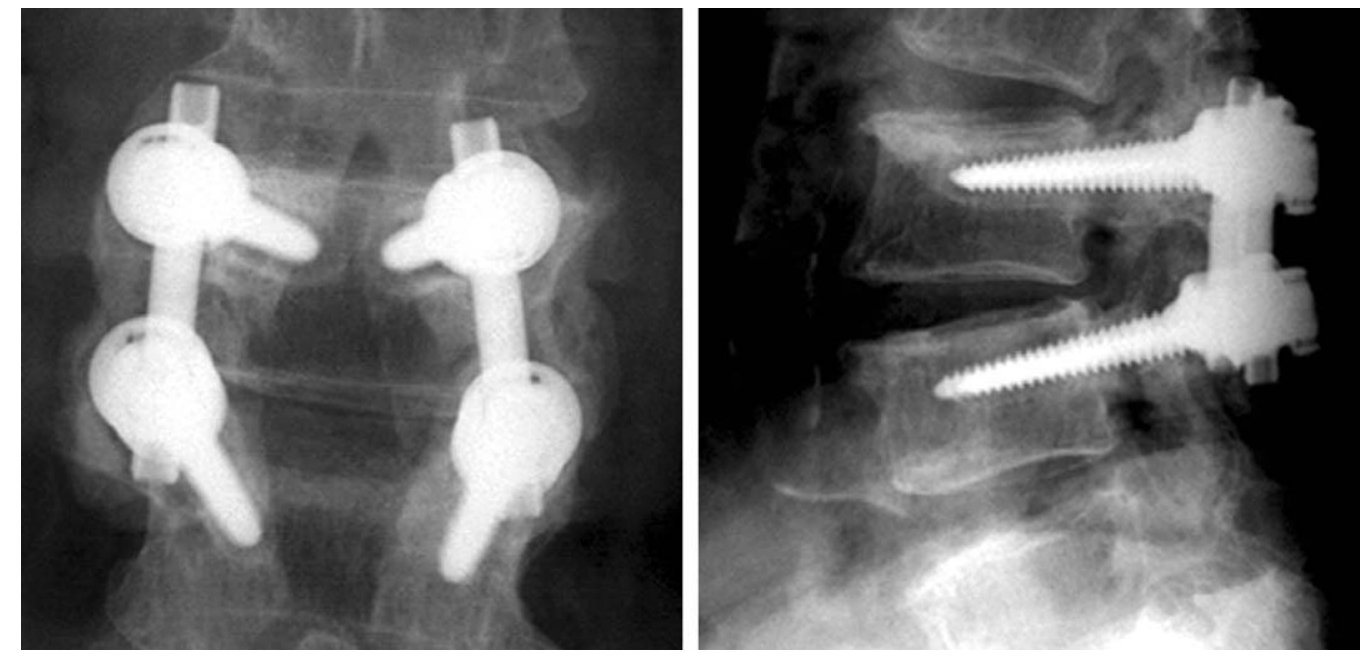

Fig. 1. Plain radiographs show the halo phenomenon (radiolucent line around the pedicle screws).
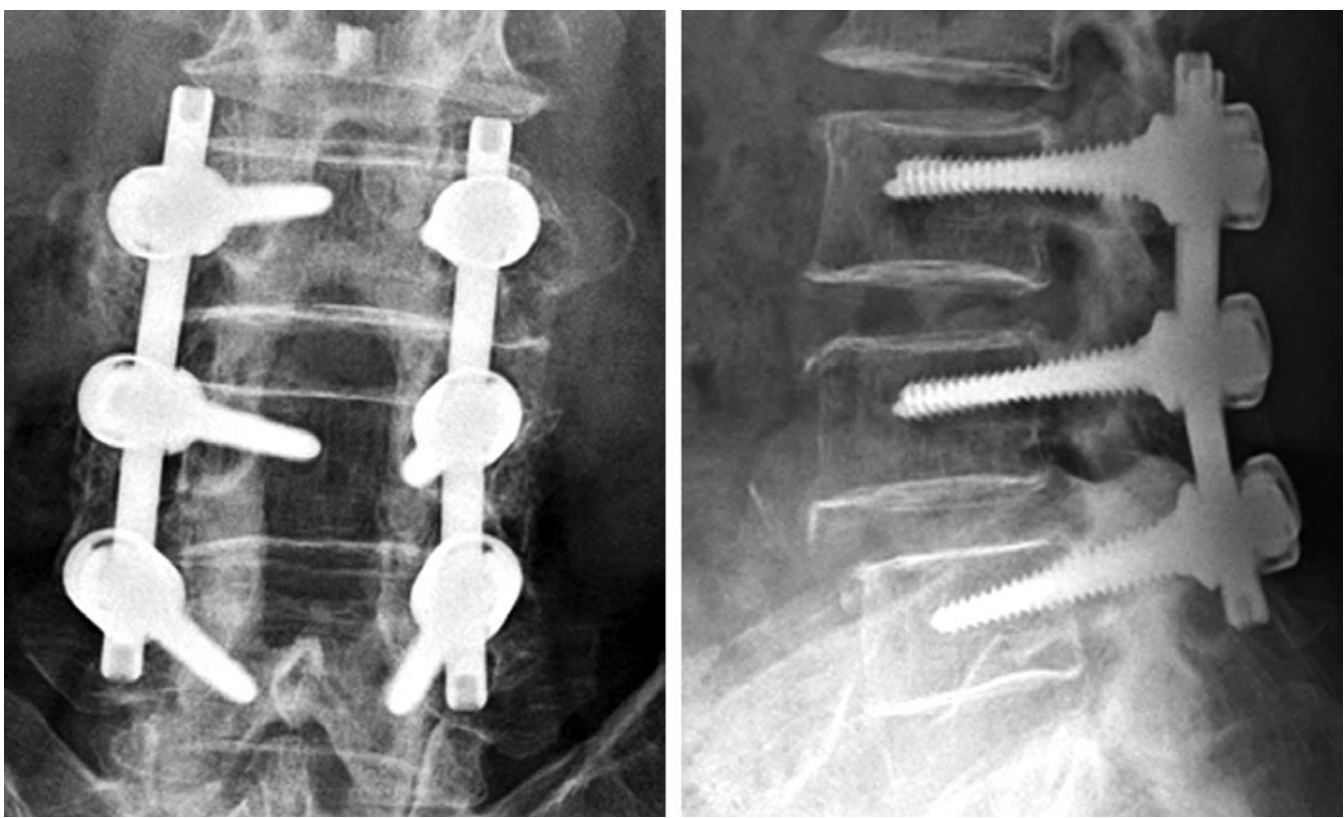

Fig. 2. Plain radiographs in a 68 -year-old female patient at postoperative month 36 . There was no halo phenomenon. 


\section{Correlation of the halo phenomenon with various factors}

In the patient group exhibiting the halo phenomenon, the mean (SD) BMD of the lumbar vertebra and the proximal femur were $0.835(0.244) \mathrm{g} / \mathrm{cm}^{2}$ and $0.361(0.131) \mathrm{g} / \mathrm{cm}^{2}$, respectively. In the group without the halo phenomenon, the mean (SD) BMD of the lumbar vertebra and the proximal femur were $0.842(0.171) \mathrm{g} / \mathrm{cm}^{2}$ and $0.442(0.157) \mathrm{g} / \mathrm{cm}^{2}$, respectively. There was no statistically significant difference between the two groups.

Variables including age, weight, menopause, BMD, osteoarthritis in the knee, VAS for back pain, VAS for leg pain, and fusion state were entered in multivariate logistic regression analysis to determine their association with the halo phenomenon in more than 2 pedicle screws. Old age, absence of osteoarthritis in the knee joint, and non-union state (the criteria of "probably not solid" or "definitely not solid") were found to be significant risk factors for the halo phenomenon in more than 2 pedicle screws $(\mathrm{p}<0.05)$. The radiological halo phenomenon did not correlate with clinical outcomes (VAS for back pain and leg pain).

\section{Discussion}

There have been many in vitro studies relating to factors affecting the stability of screws. These factors include the length of the pedicle screw, the outer diameter of the pedicle screw, the design, the compatibility with the pedicle, $\mathrm{BMD}$, and the elasticity of the cancellous bone $\mathrm{e}^{10-15}$. BMD has been regarded as the most significant factor influencing screw stability ${ }^{11,20-22}$. However, in vivo studies concerning the correlation of pedicle screw stability and BMD have demonstrated conflicting results ${ }^{23-25}$. Hence, this study was designed to determine the correlation between pedicle screw system stability and BMD, as well as other various factors.

In the current study, BMD was not related to the halo phenomenon. The BMD in the group with the halo phenomenon was no different from that in the group without the halo phenomenon. We analyzed the odds ratio for the halo phenomenon in patients with more than 2 pedicle screws with regard to age, weight, menopause, BMD, osteoarthritis of the knee, VAS for back pain, VAS for leg pain, and fusion state, using logistic regression analysis. Age, absence of osteoarthritis in the knee, and non-union state were sig- nificant risk factors for the halo phenomenon $(\mathrm{p}<0.05)$.

In elderly patients, it is thought that progression of osteoporosis, instability caused by deterioration of muscle power, and weakness in adjacent ligament force may influence the halo phenomenon. Therefore, old age could have been one of the risk factors associated with the halo phenomenon in this study. Patients without arthritis in the knee usually have higher activity in comparison with patients with arthritis in the knee, and this higher activity may be a causative factor that induces the halo phenomenon. In addition, pedicle screw instability could theoretically lead to the halo phenomenon in patients without spine fusion. However, there was no specific correlation between the halo phenomenon and surgical outcome in this study.

In the current study, several factors, such as age, arthritic changes in the knee, and fusion state influenced the occurrence of the halo phenomenon, but we believe there is no definitive evidence to suggest that these relationships have significant clinical relevance.

\section{Conclusions}

The halo phenomenon can develop after pedicle screw fixation. Age, fusion state, and osteoarthritis of the knee affect the incidence. The ultimate significance of this phenomenon is still not known, because it does not seem to influence clinical outcomes. Thus, the use of HA coating screws, expandable screws, cement augmentation, and additional surgeries to prevent the halo phenomenon is not necessary.

\section{REFERENCES}

1. Steffee AD, Sitkowski DJ: Posterior lumbar interbody fusion and plates. Clin Orthop Relat Res 1988; 227: 99102.

2. Marchesi DG, Thalgott JS, Aebi M: Application and results of the AO internal fixation system in nontraumatic indications. Spine 1991; 16: S162-169.

3. Abe E, Arai M, Sato K, et al: Pedicular screw fixation for the unstable spine. Orthop Surg Traumatol 1991; 34: 455461.

4. Zdeblick TA: A prospective, randomized study of lumbar fusion. Preliminary results. Spine 1993; 18: 983-991.

5. Essens SI, Sachs BL, Dreyzin V: Complications associat- 
ed with the technique of pedicle screw fixation. A selected survey of ABC members. Spine 1993; 18: 2231-2238.

6. Ashman RB, Galpin RD, Corin JD, Johnston CE 2nd: Biomechanical analysis of pedicle screw instrumentation systems in a corpectomy model. Spine 1989; 14: 13981405.

7. Okuyama K, Abe E, Sato K, Kamata S, Nagata A, Miyano T: Bending strength and pull-out force of pedicle screws. (in Japanese with English abstract) Orthop Surg Traumatol 1993; 36: 1357-1363.

8. Hu SS: Internal fixation in the osteoporotic spine. Spine 1997; 22: 43S-48S.

9. Law M, Tencer AF, Anderson PA: Caudo-cephalad loading of pedicle screws: mechanisms of loosening and methods of augmentation. Spine 1993; 18: 2438-2443.

10. Coe JD, Warden KE, Herzig MA, McAfee PC: Influence of bone mineral density on the fixation of thoracolumbar implants. A comparative study of transpedicular screws, laminar hooks, and spinous process wires. Spine 1990; 15: 902-907.

11. Soshi S, Shiba R, Kondo H, Murota K: An experimental study on transpedicular screw fixation in relation to osteoporosis of the lumbar spine. Spine 1991; 16: 1335-1341.

12. Carlson GD, Abitbol JJ, Anderson DR, et al: Screw fixation in the human sacrum. An in vitro study of the biomechanics of fixation. Spine 1992; 17: S196-203.

13. Okuyama K, Sato K, Abe E, Inaba H, Shimada Y, Murai H: Stability of transpedicle screwing for the osteoporotic spine. An in vitro study of the mechanical stability. Spine 1993; 18: 2240-2245.

14. Wittenberg RH, Shea M, Swartz DE, Lee KS, White AA 3rd, Hayes WC: Importance of bone mineral density in instrumented spine fusions. Spine 1991; 16: 647-652.

15. Yamagata M, Kitahara H, Minami S, et al: Mechanical stability of the pedicle screw fixation systems for the lum- bar spine. Spine 1992; 17: S51-54.

16. Lenke LG, Bridwell KH, Bullis D, Betz RR, Baldus C, Schoenecker PL: Results of in situ fusion for isthmic spondylolisthesis. J Spinal Disord 1992; 5: 433-442.

17. Kellgren JH, Lawrence JS: Radiological assessment of osteo-arthrosis. Ann Rheum Dis 1957; 16: 494-502.

18. Kim NH, Lee JW: Anterior interbody fusion versus posterolateral fusion with transpedicular fixation for isthmic spondylolisthesis in adults. A comparison of clinical results. Spine 1999; 24: 812-816.

19. Huskisson EC: Measurement of pain. Lancet 1974; 2: 1127-1131.

20. Halvorson TL, Kelley LA, Thomas KA, Whitecloud TS 3rd, Cook SD: Effects of bone mineral density on pedicle screw fixation. Spine 1994; 19: 2415-2420.

21. Bennett GJ, Serhan HA, Sorini PM, Willis BH: An experimental study of lumbar destabilization. Restabilization and bone density. Spine 1997; 22: 1448-1453.

22. Lim TH, An HS, Hasegawa T, McGrady L, Hasanoglu KY, Wilson CR: Prediction of fatigue screw loosening in anterior spinal fixation using dual energy $\mathrm{x}$-ray absorptiometry. Spine 1995; 20: 2565-2568.

23. Kumano K, Hirabayashi S, Ogawa Y, Aota Y: Pedicle screw and bone mineral density. Spine 1994; 19: 11571161.

24. Yasukawa Y, Akizuki A, Ryuzawa T, Kobayashi H, Kitahara J: Influence of BMD and BMI on the clinical results of posterolateral fusion for degenerative lumbar spondylolisthesis. J Japan Spine Res Soc 1999; 10: 254.

25. Okuyama K, Abe E, Suzuki T, Tamura Y, Chiba M, Sato K: Influence of bone mineral density on pedicle screw fixation: a study of pedicle screw fixation augmenting posterior lumbar interbody fusion in elderly patients. Spine J 2001; 1: 402-407. 